\title{
Comparison of Portable Spring Balancer with Mobile Arm Support in Aiding Self-Feeding in Quadriplegic Patients with High Cervical Injuries - Rate of Adaptation to Both Devices
}

\author{
Noriyoshi Asai ${ }^{1)}$, SAdAe Kuroiwa ${ }^{2)}$ \\ ${ }^{1)}$ Department of Occupational Therapy, School of Allied Health Science, Kitasato University, \\ 1-15-1 Kitasato, 228-0829, Japan. TEL +81 42-778-9697. \\ ${ }^{2}$ Department of Occupational Therapy, Musashi National Hospital of Psychiatry and Neurology
}

\begin{abstract}
This study aimed to identify the most effective type of upper extremity orthosis in aiding selffeeding in quadriplegic patients with high cervical injuries by comparing a portable spring balancer and a mobile arm support. A single-subject A-B-C-B-C design was used in the study, in which a subject was tested at baseline (A) followed by alternate use of a portable spring balancer (B) and a mobile arm support (C) for two rounds. Four subjects were requested to eat yogurt with the aid of one of the devices. The amount of yogurt consumed was compared between the two devices. Three of four subjects were able to eat much more the yogurt with portable spring balancer than with the mobile arm support. Only one subject used the mobile arm support more effectively than the portable spring balancer. The subjects' physical condition, specifically, muscle strength of the upper arm and tolerance appeared to be factors that influence how patients adapt to these devices. In conclusion, both the portable spring balancer and mobile arm support are effective feeding aids for quadriplegic patients with high cervical injuries. These orthoses function differently for patients with different physical characteristics.
\end{abstract}

Key words: Upper Extremity Orthosis, Quadriplegic, Feeding.

(This article was submitted Dec. 10, 1998, and was accepted Mar. 30, 1999)

\section{INTRODUCTION}

Quadriplegic patients with high cervical injuries suffer from muscle weakness and a limited range of motion. Movements such as extending their arms to reach objects and many other simple tasks that facilitate smooth daily living prove difficult or impossible to achieve. The goal of rehabilitation therefore, is to help them be independent at home and in society as a whole ${ }^{1)}$.

"Rehabilitation of a spinal cord injured patient requires that the occupational therapist, with other rehabilitation team members, help the patient achieve the highest level of independence of which he is physically capable"2). The role of the occupational therapist is to teach patients adaptive techniques, help them to acquire and learn how to use equipment, and provide consultation on physical modifications to their home or work environments ${ }^{3}$. The range of activities in daily living (ADL) is limited in patients with spinal cord injury. Orthoses and adaptive devices are useful to help them accomplish ADL, and occupational therapists are active in researching new or improved orthotic equipment which will allow quadriplegic patients to manipulate tools.

The suspension sling is a useful piece of equipment for a person with weakness of the upper arm, especially the proximal arm. The main purpose of 
this orthosis is to reduce the gravity load on the limb. This permits operational movement of the limb by muscles otherwise too weak to be functional $\left.{ }^{4}, 5\right)$. This orthosis functions to establish patterns of horizontal abduction and adduction in hand-to-body movements and hand-to-face movements, and is useful for activities such as eating, table top communication, and leisure. The disadvantage of this orthosis is the need for a large area to accommodate the suspension rod, which, being very long, precludes its use in many ordinary rooms and hospital wards. A compact form of this orthosis may be attached to a bed, wheelchair, regular chair, child's high-chair, or an overheadtrack for ambulatory patients6). King ${ }^{7)}$ has developed a wheelchair-attachable deltoid aid (compact overhead sling) designed to eliminate gravity, for use by patients with upper extremity weakness who require compact device assistance in gaining independence in daily activities. It is easily portable for use outside the home or clinical area, and requires little maintenance and virtually no adjustment. The deltoid aid is often used in the occupational therapy room to improve range of motion (ROM) and muscle strength, and serves as an aid to self-care, such as self-feeding. It functions as a suspension sling, allowing the extremity to move smoothly from table to mouth ${ }^{8)}$, and is useful for $\mathrm{C}_{5}$ quadriplegics with weak deltoids as an interim assistive aid during the acute phase of rehabilitation ${ }^{9}$.

The mobile arm support (MAS), also known as a ball bearing feeder or balanced forearm orthosis, is the most commonly used orthosis for wheelchair patients with severe proximal (shoulder and elbow) weakness or paralysis ${ }^{10)}$. The standard MAS consists of a bracket assembly or proximal ball-bearing, a proximal swivel-arm, a distal ballbearing, a distal swivel arm, a rocker arm assembly and a trough ${ }^{4}$. The MAS provides (a) support for the shoulder flexor and abduction in a sustained anti-gravity position; (b) assistance for internal rotators and abductors; and (c) assistance for internal and external rotation of the shoulder ${ }^{11)}$. It improves performance in feeding, grooming, written communication, and desk skills ${ }^{12}$. The MAS is an excellent piece of equipment, but has the disadvantages of being expensive to construct, limited in area of use, and complex to $\left.\operatorname{adjust}^{7}, 13,14\right)$. Kuroiwa $^{1)}$ has noted that patients with spasticity or rigidity cannot use the MAS smoothly, and that many spinal cord injury patients cannot control the MAS well in the initial phase of rehabilitation. To facilitate fitting the MAS, Drew et al. ${ }^{15}$ ) developed an adjustment mechanism to balance the forearm orthosis. Burt et al. ${ }^{16)}$ also designed a new linear mobile arm support to increase its functional abilities.

The portable spring balancer (PSB) was developed in Japan and has been used in hospitals and homes since 1986. People with weak upper extremities may use this orthosis as an aid for self-care activities and exercise. The mechanism of this device is similar to that of the mobile arm support and suspension sling: it supports the patient's arm during daily activities, especially eating, writing, typing, and hygiene ${ }^{17}$. This orthosis has a spring contained in an aluminum tube to eliminate gravity on the arm. It functions to assist movement in an upward direction against gravity while allowing the weight of the arm to be effective in downward motion. As a result, the quadriplegic patient can easily move his hand either upward or downward.

This study investigated whether the PSB is a more efficient to aid self-feeding in a quadriplegic patient than a mobile arm support. The efficiencies of the equipment (MAS and PSB) were measured in terms of the amount of yogurt (in grams) consumed within a specific period. A single-subject A-B-C-B-C design was used. The hypothesis under test was that the quadriplegic patient would be able to consume more yogurt within a specified period of time using the portable spring balancer than he would using a mobile arm support.

\section{METHODS}

\section{Subjects}

In accordance with Yasuda et al. ${ }^{18)}$ and Asai ${ }^{17)}$ subject selection was based on the following criteria:

1. Spinal cord injury at $\mathrm{C}_{4}-\mathrm{C}_{5}$ level

2. Passive ROM in shoulder flection and an abduction of 0-90 degrees, internal rotation of 0-90 degrees, elbow flection of 0-110 degrees, and hip flection of 90 degrees;

3. At least one muscle in the shoulder girdle, shoulder, or elbow with a grade of poor or better;

4. Stability and tolerance for wheelchair sitting for more than thirty minutes

5. No experience in using either the MAS or the PSB to aid feeding. 
The subjects available for this study were four in-patients at the National Murayama Hospital in Japan. A therapist set up the PSB and MAS for each patient, and also measured the amount of yogurt consumed. The occupational therapists were equally familiar with both types of equipment.

The four subjects were diagnosed as having spinal cord lesions at a high cervical level as a result of traumatic accidents which had taken place while playing rugby, diving, involvement in a motor accident and falling from a roof. Their characteristics, age, ROM, and muscle strength are shown in the Tables 1,2 and 3. The subjects were dependent in all activities of daily living. They had never used either a MAS or a PSB to eat by themselves, and were motivated to learn how to gain independence in feeding themselves.

\section{Apparatus}

The PSB produced by the department of the National Murayama Hospital consists of three metal parts. An aluminum tube $750 \mathrm{~mm}$ in length and 30 $\mathrm{mm}$ in diameter contains a spring $150 \mathrm{~mm}$ long and $20 \mathrm{~mm}$ in diameter to assist the arm in resisting gravity. This tube is affixed in an upright position to a table by a bracket. A specially constructed proximal arm assembly is connected to the aluminum tube by a plastic joint, thus allowing vertical movement. The third part consists of a steel bar which connects the distal arm assembly to a distal cuff which supports the arm at the elbow and wrist (Fig. 1).

The MAS used was a standard type. It consisted of a distal arm assembly, a proximal arm assembly, a trough, and a bracket. The MAS supports the weight of the arm on a ball bearing to improve its function (Fig. 2).

\section{Other Materials}

The following materials were also used in the study: an adjustable table; a plate with a special inner lip feature to keep food from sliding off when maneuvering food onto a spoon; a wrist-support splint with a palmar clip to maintain wrist exten-

Table 1. Subject Characteristics

\begin{tabular}{ccccc}
\hline Subject & Gender & Age & $\begin{array}{c}\text { Using hand } \\
\text { to hold spoon }\end{array}$ & $\begin{array}{c}\text { Duration } \\
\text { Since SCI (Week) }\end{array}$ \\
\hline 1 & Male & 18 & Left & 54 \\
2 & Male & 20 & Left & 60 \\
3 & Male & 50 & Right & 25 \\
4 & Male & 15 & Left & 15 \\
\hline
\end{tabular}

Note. $\mathrm{SCI}=$ spinal cord injury.

Table 2. Muscle Grading of Subjects

\begin{tabular}{ccccccc}
\hline Subject & Trapezius & Deltoid & $\begin{array}{c}\text { External } \\
\text { Rotators }\end{array}$ & Biceps & Supinator & $\begin{array}{c}\text { Extensor Carpi } \\
\text { Radialis Longus }\end{array}$ \\
\hline 1 & 5 & $1^{+} \sim 2^{-}$ & 0 & $2^{-}$ & 3 & 0 \\
2 & 4 & 1 & 2 & $3^{+}$ & 3 & 0 \\
3 & 4 & $3^{+} \sim 4^{-}$ & $2^{-}$ & 4 & 4 & $2^{-}$ \\
4 & 4 & $1 \sim 2^{-}$ & 0 & 2 & 3 & 0 \\
\hline
\end{tabular}

Table 3. ROM of Using Upper Extremity

\begin{tabular}{cccccc}
\hline Subject & Using & \multicolumn{2}{c}{ Shoulder } & \multicolumn{2}{c}{ Elbow } \\
& Extremity & flexion & abduction & flexion & extension \\
\hline 1 & Left & $170^{\circ}$ & $160^{\circ}$ & $140^{\circ}$ & $0^{\circ}$ \\
2 & Left & $130^{\circ}$ & $130^{\circ}$ & $140^{\circ}$ & $0^{\circ}$ \\
3 & Right & $130^{\circ}$ & $170^{\circ}$ & $140^{\circ}$ & $0^{\circ}$ \\
4 & Left & $170^{\circ}$ & $165^{\circ}$ & $140^{\circ}$ & $0^{\circ}$ \\
\hline
\end{tabular}



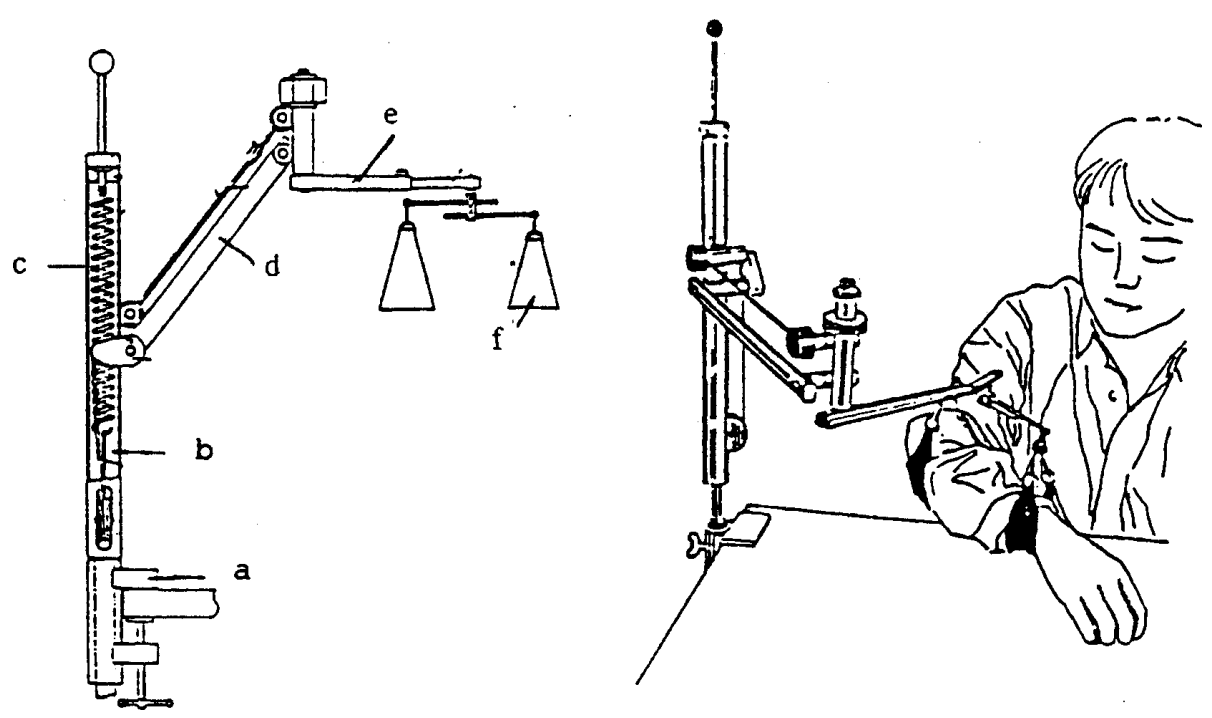

Fig. 1. A Portable Spring Balancer. (a) bracket, (b) aluminium tube, (c) spring, (d) proximal arm, (e) distal arm, (f) cuff.
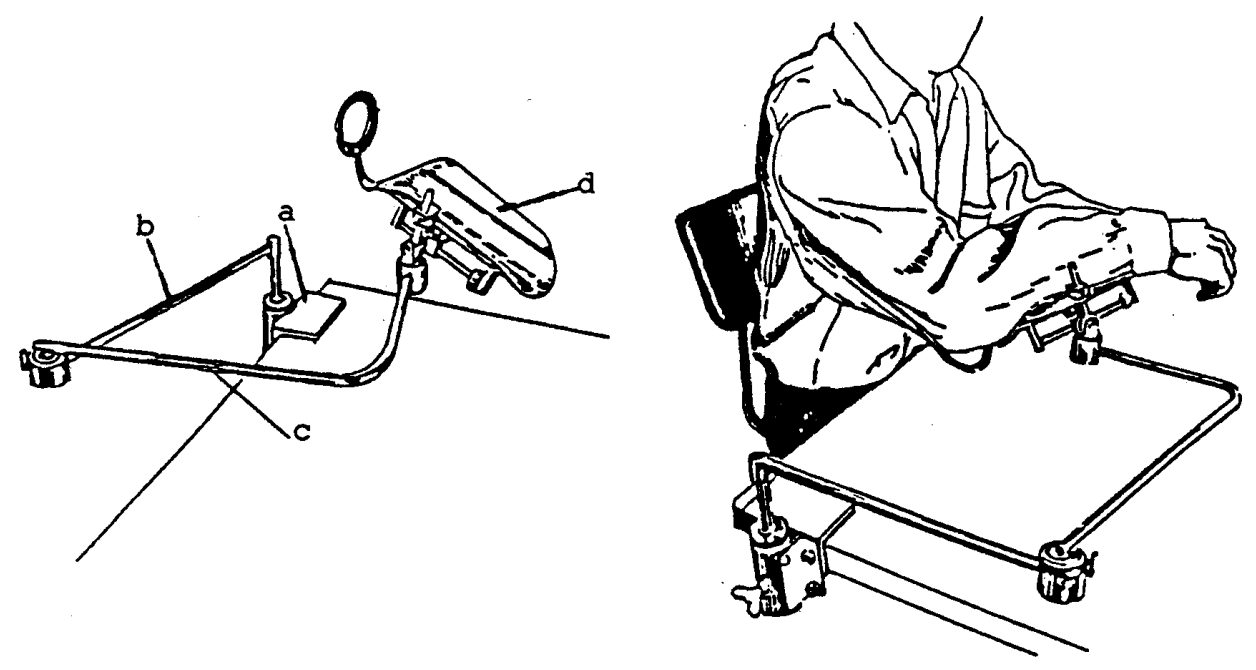

Fig. 2. Mobile Arm Support. (a) bracket, (b) proximal arm assembly, (c) distal arm assembly, (d) trough.

sion and to hold a spoon; a plastic sheet for protecting clothing; an angled tablespoon scale measuring 0-500 grams to assess the weight of the yogurt; 200 or 300 gram amounts of yogurt; and a clock.

\section{Procedure}

The study was conducted between 1991 and 1993 and the following procedure took place in the occupational therapy room at the participants' hospital. In an attempt to control external factors, measurements were taken in a quiet place at approximately the same time on the days when the subjects received occupational therapy. A singlesubject A-B-C-B-C design was used ${ }^{19,20)}$. The subject was requested to eat yogurt without an orthosis for one session to obtain baseline data (phase A). Then the patient took yogurt aided by a PSB for 8 sessions (phase B) and then switched to eating with a MAS for another 8 sessions. This was followed by another round of phase B and 
phase C. The research subjects were positioned upright and always at the same angle during examination. The PSB was mounted on a table and the MAS on the subject's wheelchair. The tablespoons were angled to facilitate eating. The subjects used a wrist-support splint with a palmar clip to maintain wrist extension and to hold the spoon. The subject in a wheelchair was positioned at the center of the table. Subject 1 was unable to conform to the protocol as he used a wheelchairmounted chin-control which interfered with the mounting of the MAS bracket on the wheelchair. Therefore, the MAS and PSB were mounted at the same place on the adjustable table. With the subjects taking yogurt with a wrist-support splint, positioning of equipment was decided taking into account ease of consumption, height of the table, position of the plate, and back angle of the wheelchair. This position was maintained throughout data collection. The occupational therapist in charge of the patient was responsible for data collection.

The plate of yogurt was set on the table. Subjects 1 and 2 were given 300 grams. Subjects 3 and 4 were given 200 grams.

The instructions were given in Japanese and they translate as, "Please eat the yogurt. You have five minutes." The occupational therapist observed and measured the amount, in grams, of yogurt left on the plate or spilled after five minutes. The result was recorded as a percentage (\%).

\section{Data Analysis}

All measurements were plotted on a graph for visual inspection for patterns that had developed over the experimental period. Upward, downward, or inconsistent patterns were noted. A celeration line was drawn to aid visual analysis of changes in the subjects' performances. The resulting celeration lines were evaluated according to the degree of change. The mean amount of yogurt consumed was calculated for each phase. These mean values were used in comparative analyses ${ }^{21}$, 22, 23). Twice a week, throughout the study, the weight of the yogurt was measured by a second occupational therapist to test inter-rater agreement. The rate of agreement was over $95 \%$.

\section{RESULT}

The measurements collected for each time point for all the subjects and the means for each phase are shown in Fig. 3. Only one measurement was obtained in phase A as baseline data, which demonstrated the inability of the subjects to eat yogurt without the aid of adaptive equipment while in a wheelchair. An inclining trend was observed in phase $\mathrm{B}$ using the PSB and in phase $\mathrm{C}$ using the MAS. Moreover, a uniform positive trend could also be observed across both $\mathrm{B}$ phases for all the subjects, showing a continuous increase in the amount of yogurt consumed using a PSB. A positive trend was also observed in the two $C$ phases except in subject 4 who was unable to eat yogurt with the MAS. This suggests a gradual improvement in task performance as the subjects became more familiar with the PSB and MAS.

Subjects were unable to eat yogurt for the first two or three times in the first $\mathrm{C}$ phase. In the $\mathrm{B}$ phases, subjects 1,3 , and 4 were able to eat yogurt from the first attempt, only subject 2 failed at the first attempt during the second B phase. Three of the four subjects consumed yogurt more easily using the PSB than the MAS.

The celeration lines for the B phases were projected into the $\mathrm{C}$ phases, and the proportion of the data points below or above these lines were evaluated. For subjects 1, 3 and 4, the data points in the $\mathrm{C}$ phases were all below the projected celeration line for the B phases. This finding suggests that the change in performance during the PSB phase was significant. Most of the data points in the $\mathrm{C}$ phase were below the celeration line, showing greater consumption of yogurt with the PSB than with the MAS. In subject 2, all the data points for the second phase $\mathrm{C}$ were above the celeration line and they all tended to reach maximum consumption. These results show that the subject was accustomed to using the MAS as a feeding device in the second $\mathrm{C}$ phase.

The mean amounts of consumption for each phase are shown in Fig. 3. In subjects 1, 3 and 4, the mean consumption for each $\mathrm{B}$ phase was larger than that in each $\mathrm{C}$ phase. The mean scores for subject 2 were not different between the first $\mathrm{B}$ phase and the first $C$ phase, but the consumption in the second $\mathrm{C}$ phase almost doubled that of the second B phase (Fig. 3).

\section{DISCUSSION}

In conducting a comparative evaluation of extremity orthoses in the clinical field, it is important 

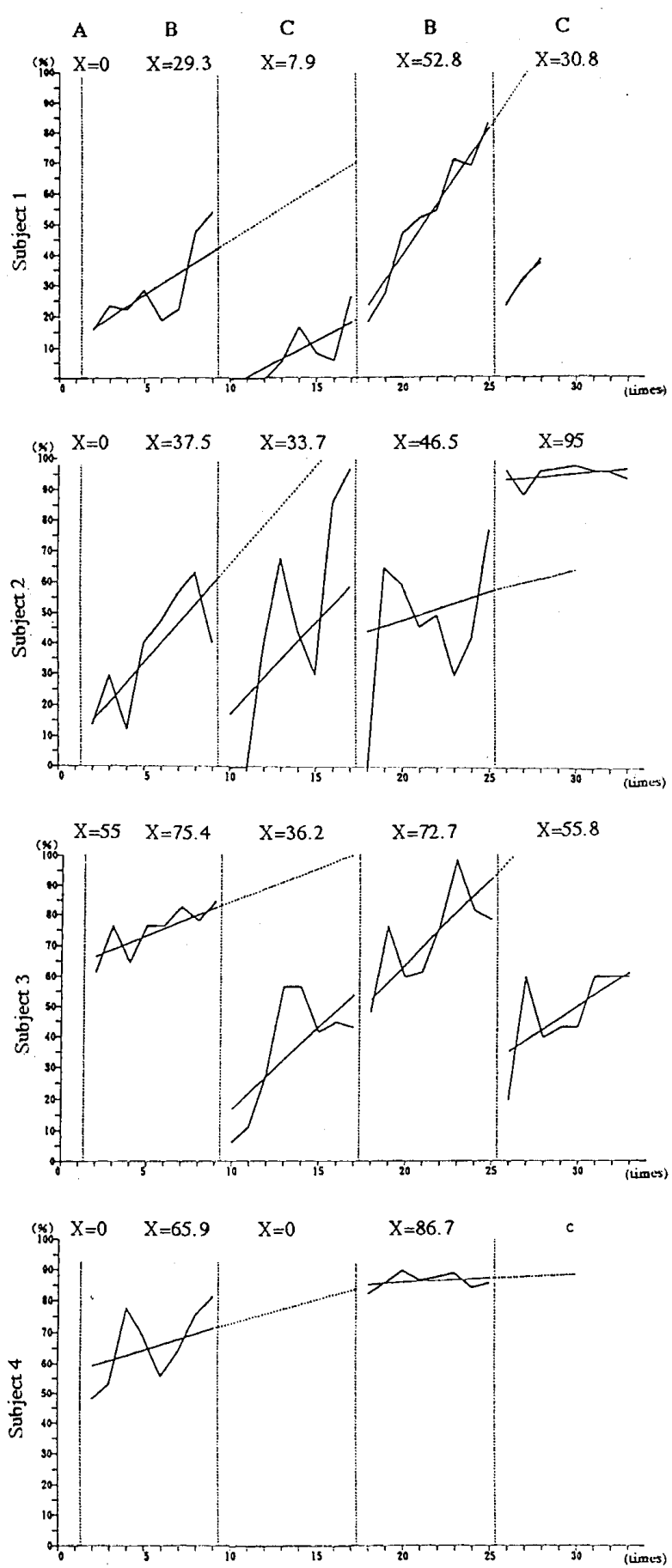

Fig. 3. The amount of yogurt consumed as percentage of total yogurt given during each phase for each subject $(\mathrm{X}=$ mean $)$.

to control all the random variables that might affect a subject's physical function, level of experience, and the examination environment itself.

Perfectly matched subjects are difficult to obtain.
A single-subject design is, however, one available method for comparing orthoses. This study evaluated the effect of using upper extremity orthoses in self-feeding on quadriplegic patients with high cervical injury using the single-subject design.

A "trend" is the direction in which a response pattern or a series of data points is progressing ${ }^{19}$ ). An inclining trend was observed in almost all of the phases for each subject. This result shows that both orthoses are relatively efficient for patients with high cervical injury. Kazdin ${ }^{24}$ ) has indicated that the magnitude of change across phases is often evident to mere visual inspection. In the present study, the magnitude was large between each B phase and $\mathrm{C}$ phase in each subject. This shows that the level of skill, rather than remaining constant, can be expected to improve with practice for both orthoses. The magnitude of changes is also examined in terms of mean yogurt consumption. Although subjects 1, 2, and 4 had the same level of spinal cord injury, they differed in the amounts of yogurt consumed when aided by the PSB or MAS.

Subjects 1 and 4 presented data in favor of the PSB in terms of the celeration lines and mean scores. They were able to use the PSB as feeding equipment more efficiently than the MAS, and consumed a greater amount of yogurt with the PSB. The relative efficiency of the PSB in these patients may be explained by the fact that both deltoids and biceps had muscle strength graded 2- in these subjects. Asai ${ }^{17)}$ has noted that having grade 2- deltoid strength is useful in triggering movement of the spring in the PSB. The power of the spring assists in elevation of the shoulder. For subject 2, the pattern in the second phase $\mathrm{C}$ showed stable maximum consumption. The mean yogurt consumption was higher in the second MAS phase than in the second PSB phase. In this subject, muscle strength was grade 1 for the deltoid, $3+$ for the biceps and 2 for the external rotator of the shoulder. The balance of his trunk was good, and his physical condition conformed to the requirements for using the MAS as reported by Thenn ${ }^{25)}$, that the patient should have at least one, and optimally two, power sources in the shoulder and/or elbow, minimally graded at fair or above; in addition it is important that patients be able to tolerate sitting in a wheel chair. The results in this study suggested that the PSB and MAS benefitted each quadriplegic patient differently. Although the level of spinal cord injury was classified $\mathrm{C}_{4}$ to $\mathrm{C}_{5}$, our subjects differed 
in their physical state such as balance of trunk, tolerance, range of motion, and muscle grading. Cusick et al. ${ }^{20)}$ indicated that validity in single-subject designs is achieved by replication of the study with a number of subjects who possess similar characteristics and who undergo similar intervention. Further experimentation on subjects with matched physical characteristics is required to confirm the present results.

\section{CONCLUSION}

A single-subject A-B-C-B-C design was used to investigate whether the PSB is more effective than the MAS in aiding self-feeding in quadriplegic patients. Both devices were found to be effective feeding aids. However, the subject's physical condition, specifically, muscle strength of the upper arm and tolerance appeared to be factors that influence how patients adapt to these devices.

\section{REFERENCES}

1) Kuroiwa $S$ : Development of portable over head arm sling and its effect of application. Study regarding to welfare equipment of development and utilization. Research Issues No. 5. pp 1-41. Ministry of Health and Welfare, Tokyo, 1981.

2) Mckenzie MW: The role of occupational therapy in rehabilitating spinal cord injured patients. American Journal of Occupational Therapy 24: 257-263, 1970.

3) Frieden L, et al: Independence: The ultimate goal of rehabilitation for spinal cord-injured persons. American Journal of Occupational Therapy, 39: 734-739, 1985.

4) Long C, et al: Upper limb orthotics. In J.B. Redford (Eds.). Occupational Therapy, J B. Lippincott Company, Philadelpia, 1986, pp 258-271.

5) Trombly CA: Orthoses: purpose and types. In C. A. Trombly (Ed.). Occupational Therapy for Physical Dysfunction, Williams \& Wilkins. Baltimore, 1989, pp 329-354.

6) Bennett RL: Orthotics for Function Part I: Prescription. Physical Therapy Review 36: 1-25, 1956.

7) King II TI: Wheelchair-attachable deltoid aid. American Journal of Occupational Therapy 37: 564-565, 1983.

8) Malick MH. Active of Daily Living and Homemak- ing. In HL. Hopkins, HD Smith (Eds.). Occupational Therapy, J B. Lippincott Company, Philadelphia, 1988, pp 258-271.

9) Sargant C, et al: Occupational therapy management of the acute spinal cord-injured patient. American Journal of Occupational Therapy 40: 333-337, 1989.

10) Haworth R, et al: Mobile arm support: An evaluation. Rheumatology and Rehabilitation 17: 240-244, 1978.

11) Chyatte SB, et al: The balanced forearm orthosis in muscular dystrophy. Arch of Phys Med \& Rehabil 60: 633-636, 1965.

12) Rogers JC, et al: Follow-up study of selfcare skills. Arch Phys Med and Rehabil 61: 316-321, 1980.

13) Yamakatu H: Occupational therapy for patients with amyotrophic lateral sclerosis. Japanese Journal of Occupational Therapy 24: 638-643, 1990.

14) Pedretti LW, et al: Mobile arm support and suspension slings, In L.W. Pedretti \& B. Zoctan (Eds.), Occupational Therapy: Practice Skill for Physical Dysfunction. The C.V. Mosby Company, Lovis, 1990, pp 397-400.

15) Drew WE, et al: Modular adjustment mechanism for the balanced orthosis. Archi of Phy Med Rehabil 60: 81, 1979.

16) Burt CM, et al: Use and prescription of linear mobile arm supports. Archi of Phy Med and Rehabil 64: 525, 1983.

17) Asai N: Development of spring balancer. Japanese Prosthetic and Orthotic Journal 2: 162-171, 1986.

18) Yasuda YL, et al: Mobile arm support: Criteria for successful use in muscle disease patients. Arch for Phy Med Rehab 67: 253-256, 1986.

19) Ottenbacher KJ: Evaluating Clinic Change, Williams \& Wilkins, Baltimore, 1986.

20) Cusick A, et al: Clinical evaluation and research in occupational therapy: Single system designs. Australia Occupational Therapy Journal 37: 31-38, 1990.

21) Barlow DH, et al: Single Case Experimental Designs. A. Wheaton, Co. Ltd, Exeter. 1984.

22) Dirette D, et al: Effects of continuous passive motion of the edematous hands of two person with flaccid hemiplegia, American Journal of Occupational Therapy 48: 403-409, 1994.

23) Wu S, et al: Effects of program on symmetrical posture in patients with hemiplegia: a single subject design, American Journal of Occupational Therapy 50: 17-23, 1996.

24) Kazdin AE: Single-case research designs. Oxford University Press, New York, 1982.

25) Thenn JE: Mobile Arm Support Installation and Use. Fred Sammons Inc, Brookfield, 1975. 